\title{
THE DEGENERATION OF PERIPHERAL NERVE FIBRES
}

\author{
BY \\ E. GUTMANN and J. HOLUBÁR \\ From the Departments of Research on the Nervous System and of Physiology, \\ Charles University, Prague
}

After section of a peripheral nerve a series of processes occurs in the distal stump involving all of its constituents and leading to a breakdown of the axons and their myelin sheaths. This process was first studied by Waller (1852) and is generally known as "Wallerian degeneration". The first part of Waller's law states that after cutting a nerve degeneration occurs in the distal stump and that degeneration is due to disconnection of the nerve trunk from its " trophic" centre, i.e., the nerve cell.

Although it is almost 100 years since this law was conceived, the mechanism of degeneration is still not clear. In order to understand the process it is necessary to investigate the nature of this " trophic influence". Only in this way can the question, Why does a nerve fibre degenerate ? be solved.

Wallerian degeneration results after a certain time in failure of transmission of nerve impulses. The time at which this failure of transmission of nerve impulses can be recorded is apparently not a constant value. It is for instance known that both the metabolic rate and temperature influence the rate of degeneration (Muralt, 1945). Additional information about the factors affecting the rate of degeneration should help in understanding the mechanism of "Wallerian degeneration".

It is necessary first to define clearly the criteria of degeneration used. After nerve section degeneration in the physiological sense appears primarily when the nerve fibre will no longer transmit impulses. As a physiological criterion we are therefore using the time at which failure of transmission of nerve impulses can be recorded. A much more complicated problem is the evaluation of the criteria of degeneration in the morphological sense. Muralt (1945) states that morphological changes generally appear only a long time after loss of excitability of the nerve fibres. The first changes in the axons, however, are observed much earlier (Ramón y Cajal, 1928) and changes in the myelin sheaths studied by the polarized light method are observed, within 24 hours of nerve section (Prickett and Stevens, 1939). The criterion of degeneration in the morphological sense will therefore be dependent on the method used. In vivo loss of continuity of the axons can on the whole be correlated to the failure of transmission of nerve impulses, and is a useful criterion of degeneration in the morphological sense. However, degenerative changes in a nerve trunk do not appear in all nerve fibres at the same time (Bethe, 1903; Weddell and Glees, 1941), and the data concerning the time at which loss of continuity of the axons can be observed have a statistical value only. Much may be expected from chemical investigations which should give valuable quantitative criteria. Most measurements, however, have been concerned with later changes after nerve section and give in fact only a measure of the time of the removal of the disintegrating myelin, and have a limited physiological significance. Changes in the lipoid content, for instance, are marked only eight days after nerve section (Johnson, McNabb, and Rossiter, 1949). Valuable quantitative criteria are given by the changes of acetylcholine content in the degenerating nerve (Muralt and Schulthess, 1944), or of cholinesterase content in the degenerating nerve (Sawyer, 1946). Data about enzyme activity in a degenerating nerve will certainly acquire great importance.

In this paper we have used physiological and morphological criteria in an attempt to study quantitatively the factors which affect degeneration of peripheral nerve fibres.

\section{Methods}

Rabbits, guinea-pigs, and rats were used in our experiments. In rabbits the peroneal, tibial and, in some experiments, the sural, nerves were used, whereas in rats and guinea-pigs the experiments were done with the whole sciatic nerve. The nerves were cut at an initial operation and, at different times after nerve section, the wounds were reopened and the nerves excised. The excised nerves were placed on platinum electrodes in a thermostat. The temperature of the chamber varied from 36 to $38^{\circ} \mathrm{C}$. but was approximately constant for any given set of observations. The electrical excitability of the nerves was tested with single condensor discharges 
through a transformer using maximal stimuli (Holubár, 1949). The electrical responses of the nerves were recorded from a cathode ray oscilloscope (highest sensitivity 80 microvolts $/ \mathrm{cm}$.) the distance between stimulating and recording electrodes being about $3 \mathrm{~cm}$. When comparing nerves from the same animal the same amplification was used for both nerves. In some experiments not only excitability but also conduction velocity was measured. In these cases photographs of the action potentials were taken and the distance from stimulus artefact to onset of the action potential was measured, the sweep of the time base being the same in both the excised nerve and the control nerve experiments. In a series of experiments the course of degeneration in vitro was followed. The excised nerves were immersed in a dish filled with $50 \mathrm{ml}$. of Krebs solution (Lundberg, 1948). The dish was put into a thermostat at a temperature of $38^{\circ} \mathrm{C}$. and continually supplied with a mixture of $95 \% \mathrm{O}_{2}$ and $5 \% \mathrm{CO}_{2}$. At different times the nerves were removed from the dish and their electrical excitability was tested. After examination the nerves were again immersed in the dish containing the Krebs solution. For histological examination the nerves were fixed in $10 \%$ formol and stained for axons (Gross Bielschowsky) and myelin sheaths (Spielmeyer).

\section{Results}

Rate of Degeneration in Rabbit, Rat, and GuineaPig.-In 31 rabbits (age 1-2 years) the peroneal nerves were cut and their electrical excitability was tested 24 to 92 hours after nerve section. Positive action potentials could be obtained for $\mathbf{7 8}$ hours after nerve section, but in three animals excitability was lost at 71,72 , and 73 hours respectively after nerve section. Excitability of the peroneal nerve in the rabbit ceases therefore about 71 to 78 hours after nerve section. Conduction velocity of the degenerating nerves was measured in 11 rabbits, and the values compared with those of the normal control side. The results can be seen in Fig. X. Conduction velocity decreases to $87 \%$ of the control. The height of the spike potential also decreases progressively ; it was $71 \%$ and $95 \%$ after 30 hours, $79 \%$ after 43 hours, $30 \%$ after 48 hours, and $6 \%$ after 62 hours. In the last case the fastest nerve fibres were still conducting with a velocity of $87 \%$ of the control side. Such a finding indicates a sudden loss of excitability of nerve fibres as suggested by other authors (Rosenblueth and Dempsey, 1939 ; Erlanger and Schoepfle, 1946). To test the assumption of a centrifugal course of degeneration (Rosenblueth and Dempsey, 1939; Parker, 1933) excitability was tested in the proximal and distal part of the sectioned nerve trunk. Excitability was found to be the same in both parts of the nerve trunk. In no case did excitability of the proximal half disappear before that of the distal one. Failure of transmission, therefore, appears to set in simultaneously in all parts of the degenerating nerve (Erlanger and Schoepfle, 1946).

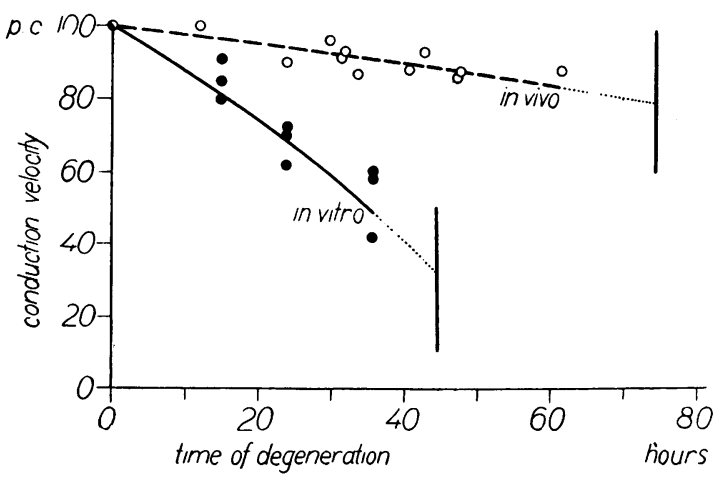

FIG. X.-Conduction velocities in nerve fibres degenerating in vivo and in vitro, at different times after section of the peroneal nerve in the rabbit. Conduction velocities are expressed as percentages of their initial values. The vertical lines indicate the time at which approximately failure of transmission of nerve impulses is recorded.

The interval at which transmission of nerve impulses fails after nerve section was also determined in the sciatic nerves of rats and guinea-pigso The results were similar to those obtained in rabbitso In 31 rats the results were as follows: Action potentials after nerve section: 72 hours, + 흥 78 hours, + ; 79 hours,,+- ; 80 hours, + , $+\frac{6}{2}$. ,,,,,,,,$++-------; 81$ hours,,,$++-;$ ,,,$----; 82$ hours,,,,,,------ ; 96 hours, - , - In nine guinea-pigs the results were as follows : 66 hours, + ; 68 hours, + ; 72 hours, - ; 72 hours, - ; 74 hours, + ; 77 hours, $+; 78$ hours, $+; 82$ hours, - ; and 83 hours, - -. The greater scatter of these data may have been due to the difficulty of obtaining animals of similar age.

Histological Changes after Nerve Section in Rabbit.-Histological changes in the peripheral nerve fibres after nerve section have been described in great detail (Ramón y Cajal, 1928; Nageotte, 1922), but apparently no systematic study correlating physiological and morphological data has been undertaken. Forty hours after section of the peroneal nerve in the rabbit (nerve excitable, conduction velocity almost unaltered) visible changes can be observed in the nerve fibres. In all of the nerve fibres continuity of the fibres is maintained, but they show irregular outlines (Fig. 1). There are fusiform swellings of the axons at places with a thin connecting strand of axoplasm. Forty-eight hours after nerve section (nerves excitable) the continuity of the nerve fibres is still maintained (Fig 2). 

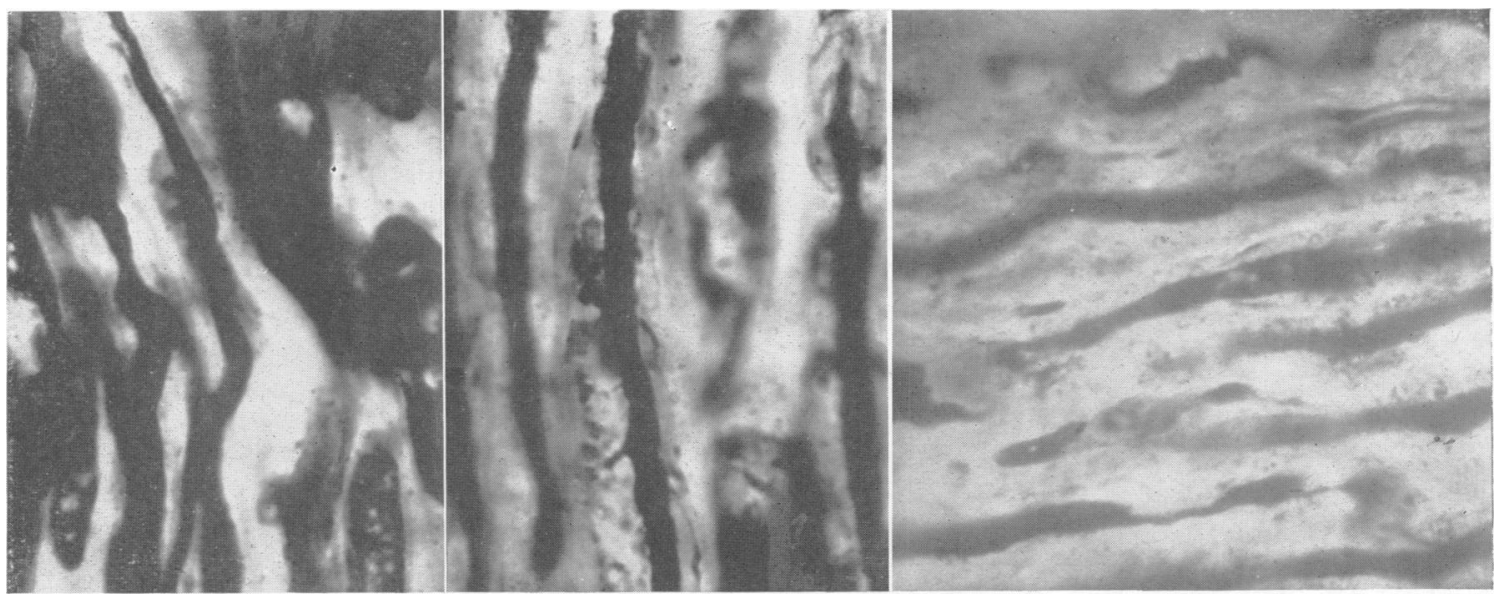

Fig. 1.-Axons in the peroneal nerve 40 hours after section. Nerve excitable. (Gross Bielschowsky, $\times 750$.)

FIG. 2.-Axons in the peroneal nerve 48 hours after section. Nerve excitable. (Gross Bielschowsky, $\times 750$.)

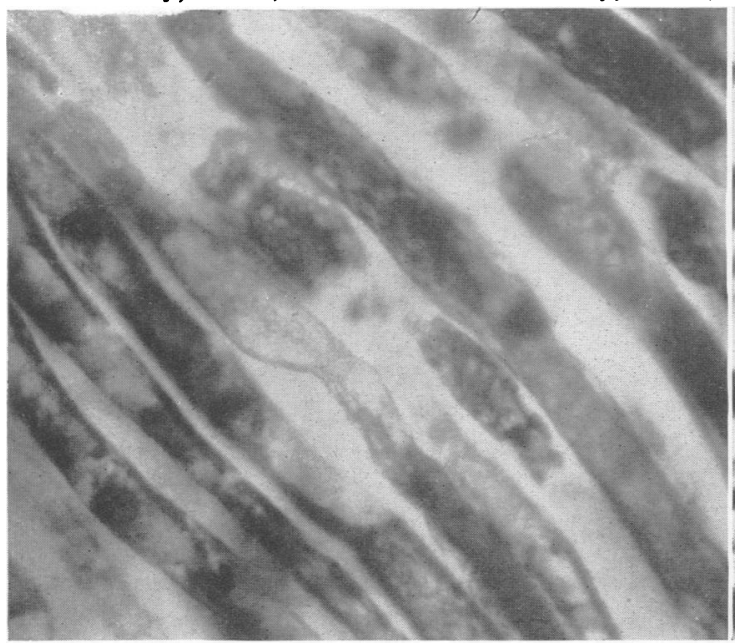

Fig. 4.- Myelin sheaths of fibres in the peroneal nerve 72 hours after section. Nerve not excitable. $($ Spielmeyer, $\times 380$.)

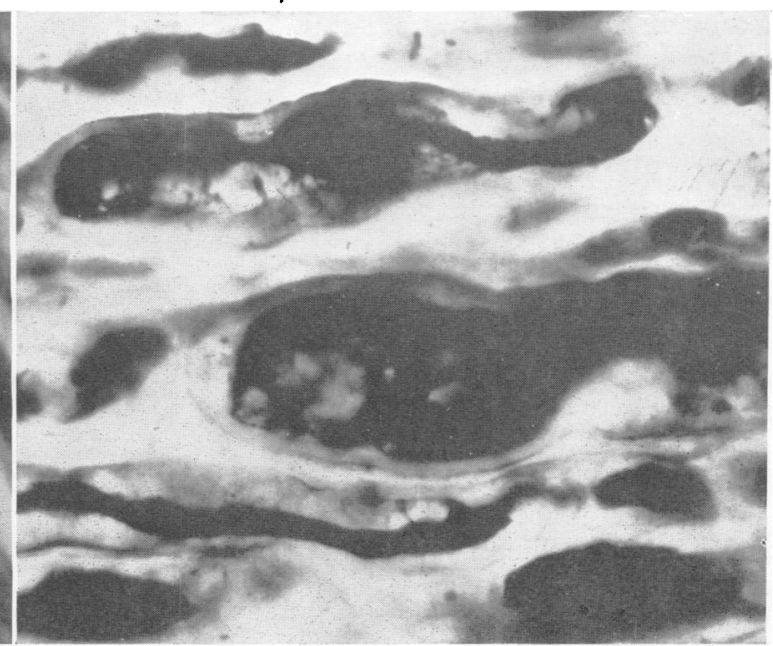

Fig. 5.-Axons in the peroneal nerve 86 hours after section. Nerve not excitable. (Gross Bielschowsky, $\times$ 750.)

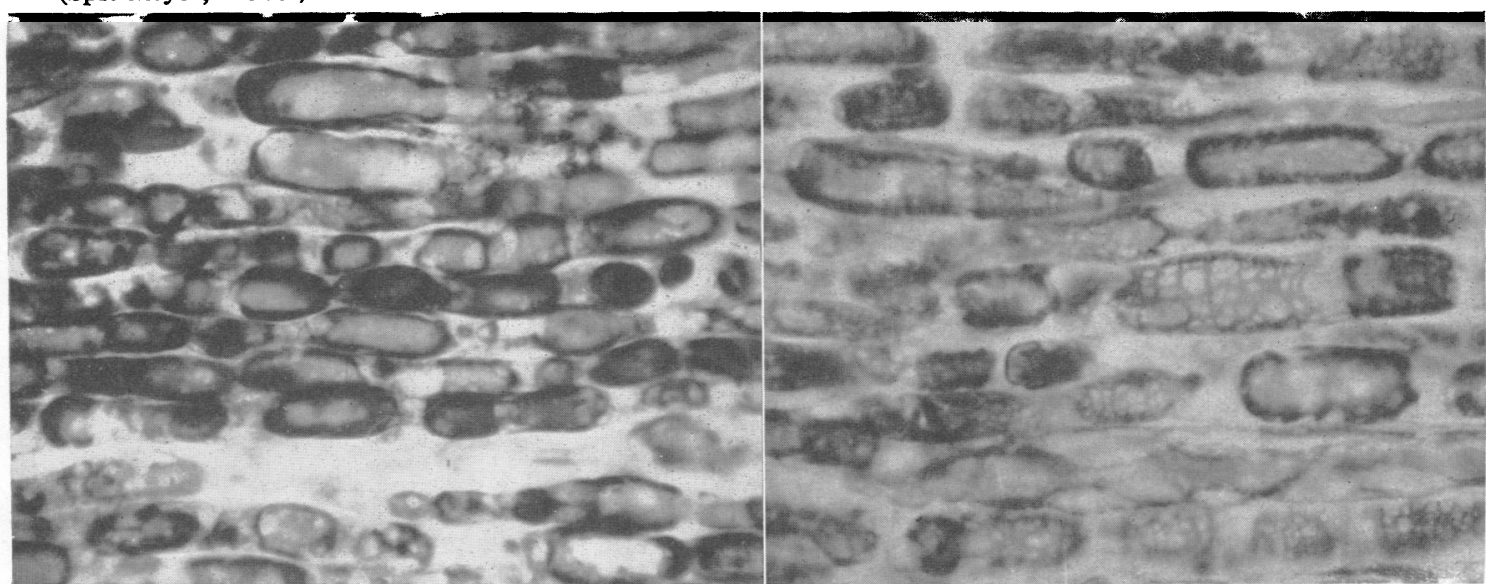

Fig. 6.-Myelin sheaths of fibres in the peroneal nerve 7 days Fig. 7.-Myelin sheaths of fibres in peroneal nerve after section. (Spielmeyer, $\times 380$.) 72 hours after homoeografting. (Spielmeyer, $\times 380$.)

IG. 3.-Axons in the peroneal nerve 72 hours Bielschowsky, $\times 750$.)

$\vec{\circ}$ 
Sixty hours after nerve section (nerves excitable, small decrease of conduction velocity only) almost all of the axons have an unbroken outline but in a few fibres of small diameter the axons are broken down into segments. In many fibres the myelin shows an unduloid outline, and the myelin is drawn apart at the nodes. Seventy hours after nerve section (nerves excitable; small decrease in conduction velocity; considerable decrease of the height of the action potential) continuity of most of the axons is intact but in a few, breakdown of continuity can be observed. The myelin sheaths are swollen but no ovoid formation can be observed. Seventy-two hours after nerve section (nerve not excitable), most of the axons are breaking down into segments, but there is still continuity of a few fibres, especially the thicker ones. The axons are swollen. Often there is only a thin strand of connecting axoplasm and breakdown of continuity occurs often at the nodes (Fig. 3). In some fibres there is considerable retraction of the myelin at the nodes of Ranvier, but only in a very few fibres can breakdown of the myelin sheath into segments be observed (Fig. 4). Eighty-six hours after nerve section, all of the nerve fibres show breakdown of continuity of the axons. Some of the large myelinated fibres are found in large digestive chambers which contain small twisted segments or even granular fragments of axonic material (Fig. 5). Seven days after nerve section the axons show a similar picture. They are broken down into small segments or granules. Some tubes are empty for longer stretches. The myelin sheaths are now breaking down into ovoids and smaller globules, but some sheaths still show continuity (Fig. 6).

Degeneration does not progress in all nerve fibres at the same rate, and it appears that in general the small medullated fibres are affected before the large ones. However, with the failure of transmission of nerve impulses, breakdown of the axons into segments can be observed in almost all the nerve fibres. At this time many myelin sheaths still show continuity, and apart from some swelling there are no certain changes. Loss of continuity of the axons is therefore a useful index of degeneration in the morphological sense, and can be correlated with the physiological failure of transmission of nerve impulses.

Effect of Age on Rate of Degeneration.-In five 2-month old rabbits the peroneal nerves were cut at an initial operation. Forty-eight, 66, 68, and 70 hours later the nerves were excised and their excitability was tested. Failure of transmission of nerve impulses was recorded between 66 to 68 hours after nerve section. In rabbits of 1 to 2 years of age excitability disappeared between 71 to 78 hours after nerve section. It appears therefore that the rate of degeneration decreases with age, and this finding often explains the individual variations found when testing the rate of degeneration of different animals.

Rate of Degeneration of Motor and Sensory Nerve Fibres.-Preliminary results of these experiments have already been reported (Gutmann and Holubár, 1949). The sural nerve - a purely sensory nerve-and the peroneal-a mixed nerve-were used in these experiments, and comparison was made always between the nerves of the same animal. Thirty-one 1 to 2-year-old rabbits were used. In the sural nerve conduction failed after about 63 to 68 hours, and in the peroneal nerve after about 72 to 80 hours after nerve section. It appears therefore that sensory nerve fibres degenerate earlier than the motor nerve fibres. The height of the action potential decreases with an increasing time of degeneration, and this occurs more quickly in the sensory nerve fibres. Fig. $Y$ shows the action potentials from the peroneal (A) and the sural (B and C) nerve immediately and 48 hours after nerve section. During this time the height of the action potential in the peroneal nerve had decreased by $50 \%$. In the sural nerve the decrease of the height of the action potential is much more marked $(98 \%)$. However, the $\mathrm{C}$ spike is, though much smaller, still larger than the A spike. This finding would suggest that some unmedullated fibres are relatively more resistant than the A fibres (Weddell and Glees, 1941).

Rate of Degeneration in Unconnected Nerve Fibres.-Another factor affecting the rate of degeneration might be the vascularization of the interrupted nerve trunk. This factor could to some extent be studied in unconnected degenerating nerve fibres, i.e., nerve trunks which were cut at two levels, high and low. In this way part of the blood supply was interrupted. In 16 rabbits the peroneal nerves were cut high up in the thigh on both sides, but also lower down at the knee on one side only. The wounds were mostly reopened shortly before the time at which failure of transmission of nerve impulses could be expected. When recording the electrical responses the distance between stimulating and recording electrodes was the same in both nerves and the same amplification was used for both nerves of the same animal. In the distally connected nerves the height of the action potentials were expressed as percentages of those of the unconnected (i.e., twice-sectioned) nerves. After 24 hours it was $95 \%$ and $58 \%$; after 48 hours, $47 \%$ and $33 \%$; after 65 hours, $69 \%$; after 66 hours, $33 \%$ and $32 \%$; 

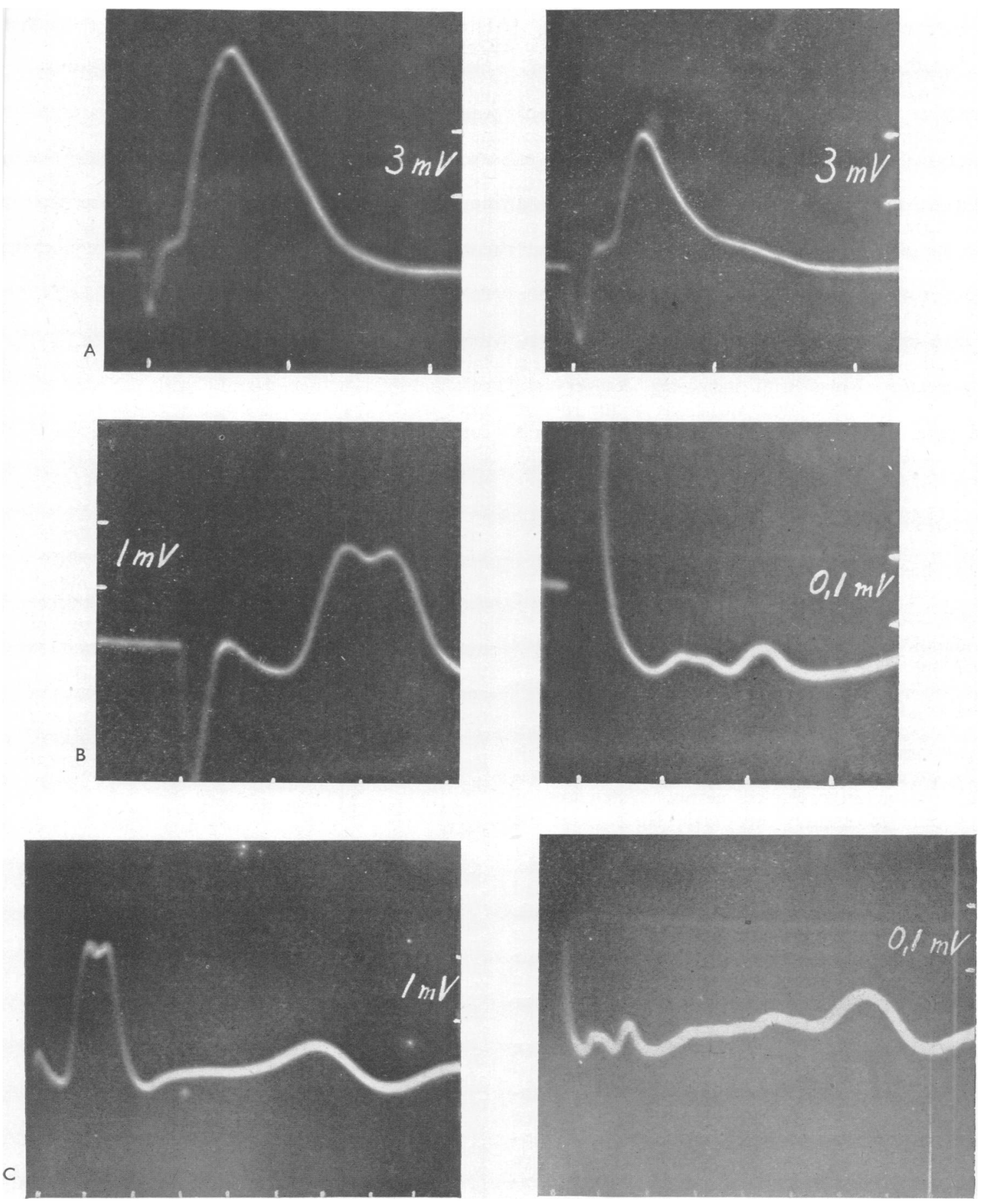

Fig. Y.-Action potentials of the peroneal (A) and the sural (B and C) nerves of the control side (left), and 48 hours after section (right). Fig. C shows the action potential of the sural nerve at a slower time sweep, thus recording the $\mathrm{C}$ spike. Forty-eight hours after nerve section, the height of the action potential in the peroneal nerve is $50 \%$ of the control side ; the decrease of the height of the action potential in the sural nerve is much more marked. In the normal sural nerve the $A$ spike is three times larger than the $C$ spike; 48 hours later the $C$ spike is twice as large as the A spike. (Time $0.4 \mathrm{~m} / \mathrm{sec}$.) 
after 70 hours, $46 \%, 15 \%$, and $47 \%$; after 71 hours, $75 \%$ and $7 \%$; after 72 hours, $71 \%$; and after 73 hours, $25 \%$. Failure of transmission of nerve impulses in the unconnected (i.e., twicesectioned nerve) was never recorded before that of the connected (i.e., once-sectioned nerve) one. Indeed, in two cases failure of transmission could be recorded (at 72 and 73 hours after nerve section) in the connected nerve, while in the unconnected nerve the amplitude was still relatively high (about $400 \mu \mathrm{V}$ ). The height of the spike in the periods shortly before failure of transmission of nerve impulses was always greater in the unconnected nerve, but this relation did not show a systematic change with increasing degeneration. However, the individual variations of the rate of degeneration are considerable in different animals and such a correlation, if it existed, could probably only be detected in the nerve of one animal.

Rate of Degeneration in Autotransplanted Nerves. -In five rabbits the peroneal nerve was cut twice, i.e., high and low, and completely isolated and inserted freely between the muscles. The degenerating nerves were thus without any blood supply. Forty-eight, 66, and 70 hours (three cases) after nerve section the wounds were reopened and the nerves tested for excitability. Action potentials were obtainable 48, 66, 70, and 70 hours after nerve section. Only in one case ( 70 hours after nerve section) was failure of transmission of nerve impulses recorded. There is therefore no certain evidence of quicker degeneration in autotransplanted nerves. Differences, if present, are certainly very small. In one case the peroneal nerve was transplanted subcutaneously, and 48 hours later action potentials were obtainable. In another case the peroneal nerve was transplanted intraperitoneally, and 48 hours later it was possible to obtain action potentials, though the height of the action potential was smaller than on the control side where degeneration in the connected peroneal nerve was tested. It is therefore probable that under these latter unfavourable conditions degeneration takes place at a quicker rate. though the difference does not seem to be very marked.

The observation that degeneration in autotransplanted nerve fibres does not proceed at a quicker rate suggests that the disturbance of vascularization which is produced by isolating and twice cutting the nerve does not affect the rate of degeneration. Further, histological preparations taken from autotransplanted nerve fibres sectioned three days previously, show no certain differences from those taken from degenerating nerve fibres with their end organs connected. Seventy-two hours after nerve section the axons show breakdown of their con- tinuity, the myelin sheaths show early ovoid formation in some fibres and may have a flaky appearance with irregular vacuolization. Seven days after nerve section there is a complete breakdown of the axons; the myelin sheaths show typical ovoid formation, but also bad staining and a flaky appearance. Up to three days after nerve section, the histological picture of degeneration in unconnected autotransplanted nerve fibres is not much different from that in connected nerve fibres. The flaky appearance and poor staining of the myelin sheaths seems to be the only difference, and this is more apparent seven days after nerve section. At this stage there is also necrosis of many Schwann cells and intra- and epi-neural fibrosis. No doubt, these segments devoid of blood supply will in due course be broken down by the phagocytolytic action of mesenchymal elements. This study, however, is not concerned with the ultimate fate of such tissue in the organism.

Rate of Degeneration in Regenerating Nerve Fibres.In some of the previous experiments it was shown that motor nerve fibres degenerated more slowly than sensory ones. Motor nerve fibres are thicker than are sensory, and it appeared that thickness of myelin sheath is an important factor in determining the rate of degeneration after nerve section. It appeared therefore important to test this factor on nerve fibres which under certain conditions have a small diameter of axons and myelin sheaths. Regenerating nerve fibres were therefore chosen as they are thin and attain a progressively larger diameter during regeneration (Gutmann and Sanders, 1943).

In four rabbits the peroneal nerves were crushed on one side in the middle of the thigh. Two months later the peroneal nerves were cut on both sides just above the crush on the previously operated side. In one animal the wound was reopened 24 hours later and the nerves were tested for excitability. Excitability on the previously crushed side was still present, the height of the action potential being, however, very small $\left(8 \frac{1}{2} \%\right.$ of the control side). In three animals the nerves were excised 48 hours after nerve section. Failure of transmission of nerve impulses could be observed in all cases. Regenerating nerve fibres undergoing degeneration at this time had already lost their excitability, whereas almost normal excitability could be observed at the control side. Regenerating nerve fibres degenerate therefore at a quicker rate, the latter being perhaps dependent on the small diameter of the nerve fibres during regeneration.

Rate of Degeneration in Central Stump of a Sectioned Nerve Trunk.-In a preliminary report 
(Gutmann and Holubář, 1948) we have shown that considerable changes are taking place in the central stump of a sectioned nerve. Six months after nerve section there is a decrease of conduction velocity by $30 \%$, of the amplitude of the action potential by $50 \%$, and of the thickness of the myelin sheath by $27 \%$. These changes in the central stump of a sectioned nerve are apparently the result of relative inactivity. According to the relation between conduction velocity and thickness of a nerve fibre (Erlanger and Gasser, 1937; Grundfest, 1940) there is also a corresponding decrease of conduction velocity. Cutting the nerve fibres produces therefore thinner myelin sheaths and in such fibres of the central stump the rate of degeneration was measured.

In three rabbits the peroneal nerve was cut and resected at the knee to make reinnervation of the peripheral stump impossible. Six months later a reoperation was performed, and the central stump of the sectioned peroneal nerve was cut high up in the thigh (thus causing degeneration in an unconnected segment of nerve), whereas on the control side the normal peroneal nerve was cut twice (i.e., high up in the thigh and lower down). In one animal the nerves were excised 24 hours later, and their excitability was tested. Action potentials in the sectioned central stump were obtained, but the height of the action potential was considerably smaller than on the control side $(25 \%$ of the control side). In the remaining two animals the nerves were excised 48 hours after section. In the sectioned central stump failure of transmission was recorded whereas normal excitability was recorded on the control side.

In three animals the peroneal nerve was cut on one side and the reoperation at which the peroneal nerves were cut on both sides (high and low on the control side, high on the previously operated side) was performed one year later. Twenty-four hours later the sectioned central stump was still excitable, but the height of the action potential was only 50 per cent. of that of the control side. In the second animal excitability was tested 32 hours after section. The sectioned central stump was excitable, but the height of the action potential was now only $7 \%$ of that of the control side. In the third animal excitability was tested $\mathbf{4 8}$ hours after section. The sectioned central stump was no longer excitable, whereas almost normal excitability was recorded on the control side.

Therefore, nerve fibres in the central stump of a sectioned nerve, which have thinner myelin sheaths, also degenerate at a quicker rate. These experiments provide further evidence of the effect of thickness of the myelin sheath on the rate of degeneration.
Degeneration in Homoeotransplanted Nerves.-In seven rabbits the peroneal nerves were cut, isolated, and resected and transplanted between the muscles of the thigh of another rabbit. Twenty-four, 38, and 44 hours later the wounds were reopened, when the homoeotransplanted nerves were still found to be excitable. In four animals in which excitability was tested $\mathbf{4 8}$ hours after grafting failure of transmission was demonstrated. Homoeografts, therefore, lose excitability quicker than autografts. In similar experiments undertaken in rats, the homoeografts again. lost excitability about $\mathbf{4 8}$ hours after grafting.

Histological preparations taken from these free homoeografts show the following changes. Twentyfour hours after grafting, axons and myelin sheaths show no certain differences to those of the autografts. The Schwann cells, however, show irregular outlines, and some of them pyknosis. There is considerable infiltration of leucocytes and lymphocytes, especially epineurally, but also at some places intraneurally. Forty-eight hours after grafting a few axons show breakdown of continuity. Many axons show marked fusiform swellings with thin connecting bridges, and it seems that these changes are at this interval more marked than in autografts. The myelin sheaths show no changes different from those of autografts. Many Schwann cells have a pale cytoplasm ; some are pyknotic and irregular in outline. There is a massive infiltration of lymphocytes and leucocytes (Fig. 8), especially in the perineurium. Seventy-two hours after grafting (Fig. 7) there is a complete breakdown of the axons, with the myelin sheaths breaking down into segments ; this myelin change seems to be more advanced than in autografts.

On the whole the histological differences between homoeografts and autografts as regards the breakdown of axons and myelin sheaths, is small. The small degree of breakdown of axons 48 hours after grafting cannot be correlated to the quicker failure of transmission of nerve impulses. It is possible that the early degenerative changes in the Schwann cells and the considerable small cell infiltration are related to the quicker loss of excitability.

Rate of Degeneration in Heterotransplanted Nerves.-In nine rabbits the peroneal nerves were removed and grafted between the muscles of the thigh of rats. Eight, 10, 15, and 24 hours later the heterografted nerves were tested for excitability. The results were as follows: Action potentials after 8 hours,,++ ; after 10 hours,,+- ; after 15 hours,,,,---- ; and after 24 hours, - .

In six rats the sciatic nerves were removed and grafted between the muscles of the thigh of rabbits. The results were as follows : action potentials after 
15 hours, +, -, - ; after 20 hours, -, - ; and after 24 hours, - .

Failure of transmission of nerve impulses in heterografts is therefore recorded at an earlier time (i.e., about 15 hours after grafting) than in homoeografts.

Histological preparations from the heterografts showed the following changes. Eight, 15, and 24 hours after heterografting the axons and myelin sheaths show no sign of disruption of continuity. Twentyfour hours after grafting the Schwann cells show a pale cytoplasm, granular and pyknotic nuclei, and there is a considerable small cell infiltration in the perineurium as well as between the nerve fibres. Forty-eight hours after grafting the majority of the axons show intact continuity: the axons are swollen and show at places irregular and poor staining. A few axons are breaking down into cuboid fragments. The same appears to occur in a few myelin sheaths. There is a massive small cell infiltration (Fig. 9). In heterografts three and four days old continuity of axons and myelin sheaths are mostly preserved, but in some axons there is breakdown to short twisted and sometimes cuboid segments (Fig. 10). Also some myelin sheaths show definite breakdown into ovoids (Fig. 11). Schwann cells are difficult or impossible to identify ; many are pyknotic or necrotic and there is a marked small cell infiltration. (The early changes in Schwann cells in homoeo- and heterografts will be described in a separate paper.) In seven-day-old heterografts the majority of the axons and myelin sheaths shows still intact continuity, so that in heterografts even at this interval "degeneration" does not occur in the same way as in homoeo- and autografts. What is more, the failure of transmission of nerve impulses occurs, unlike autografts, at a time when there is still continuity of the axons. Other factors leading probably to anoxia of the nerve fibres must be responsible, and it is possible that during homoeo-, and especially hetero-, degeneration the Schwann cells quickly cease metabolic activities and that this affects transmission in the nerve fibres.

Degeneration of Nerves in vitro.-The foregoing experiments have shown that different factors affect the rate of degeneration of nerve fibres. However, the in vivo testing of the excitability of nerve fibres has the disadvantage that the nerve fibres can be examined only once. It was therefore thought that observations on degeneration of nerve fibres in vitro might offer a method which would allow of testing a nerve fibre repeatedly during a longer time. Moreover, physiologists often do not realize that when testing electrical characteristics of excised nerves during a longer time (as is often done in pharmacological experiments) they are in fact performing experiments on nerves degenerating in vitro.

Histological changes in nerve fibres kept in Ringer solution at $38^{\circ} \mathrm{C}$. were observed by Feiss and Cramer (1913), but no systematic electrophysiological data seem to be available. In three rabbits the peroneal nerves were excised and immersed in a dish of Ringer solution kept at $38^{\circ} \mathrm{C}$. in a thermostat. Failure of transmission of nerve impulses could be observed one to two hours later. Histologically these nerves show, of course, no signs of degeneration. Loss of excitability is apparently due to anoxia. Experiments were therefore undertaken in 15 rabbits, and the nerves were immersed in a dish of Krebs solution at $38^{\circ} \mathrm{C}$., which was supplied continuously at a rate of $0 \cdot 1$ to 0.2 litres per minute, with a gas mixture of $95 \% \quad \mathrm{O}_{2}$ and $5 \% \quad \mathrm{CO}_{2}$.
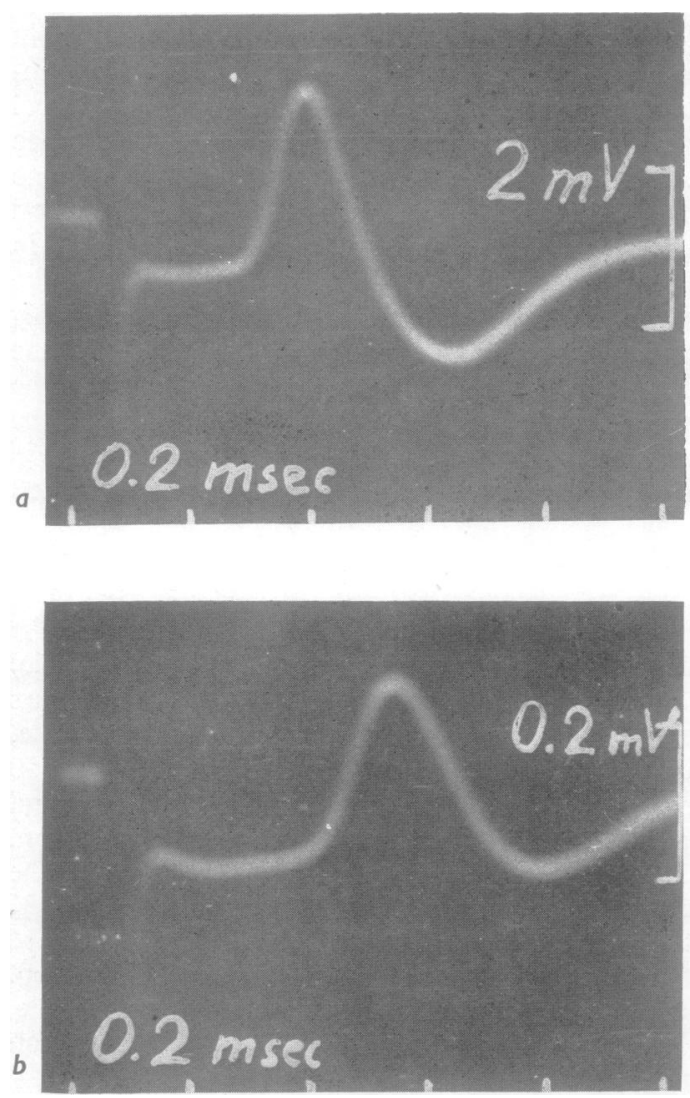

FIG.Z.-Action potentials of the peroneal nerve of the rabbit (a) immediately after excision of the nerve (conduction velocity $70 \mathrm{~m} / \mathrm{sec}$.), (b) 26 hours after degeneration in vitro (conduction velocity $42 \mathrm{~m} / \mathrm{sec}$.), two hours later the nerve was no longer excitable. 


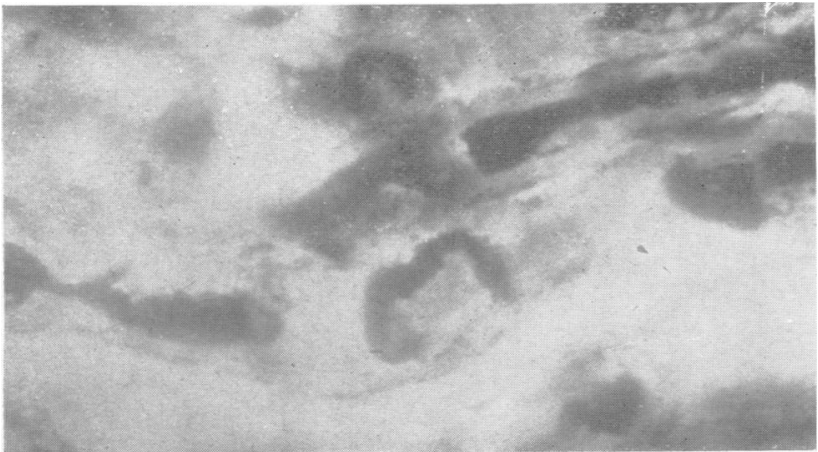

FIG. 10.-Axons of a peroneal nerve four days after heterografting. (Gross Bielschowsky, $\times 750$.)

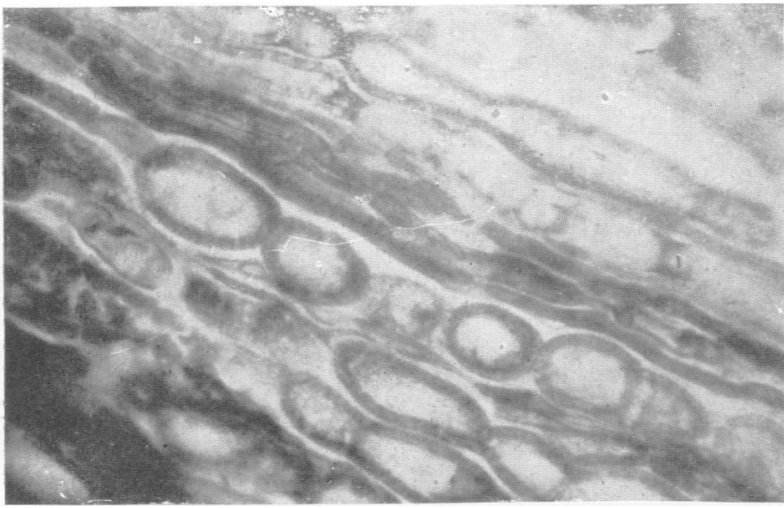

FIG. 11.-Myelin sheaths from the paroneal nerve four days after heterografting. (Spielmeyer $\times 380$.)

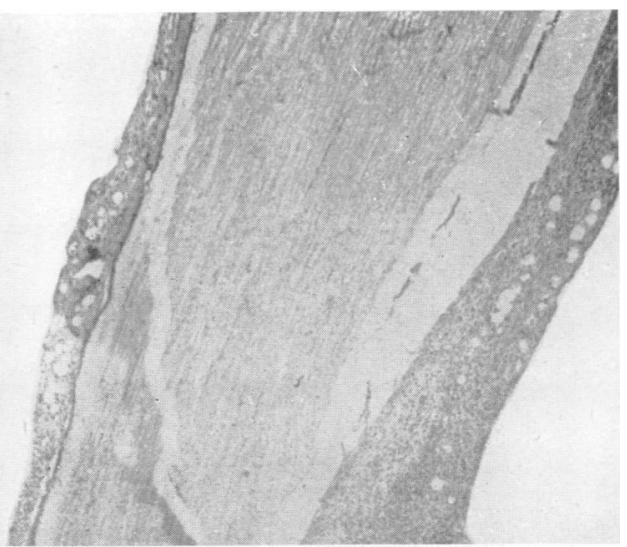

FIG. 8.-Peroneal nerve 48 hours after homoeografting. (Hæmatoxylin and cosin, $\times 30$.)

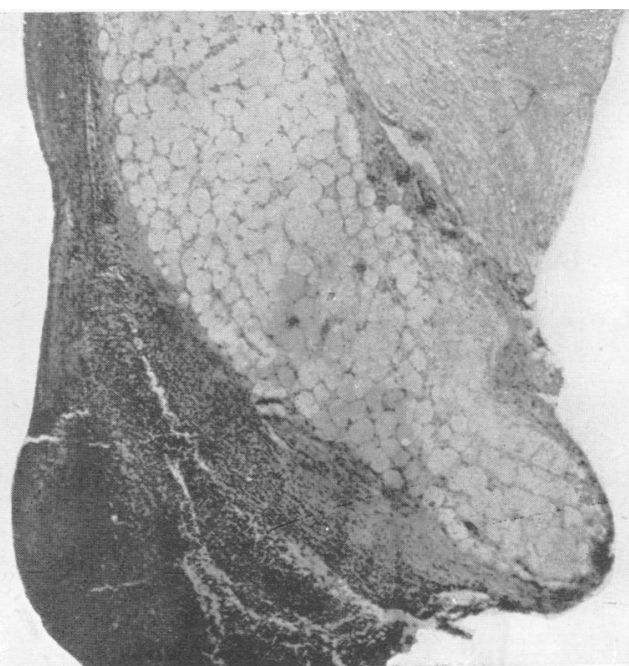

Fig. 9.-Peroneal nerve 48 hours after heterografting. (Hæmatoxylin and eosin. $\times 30$.)

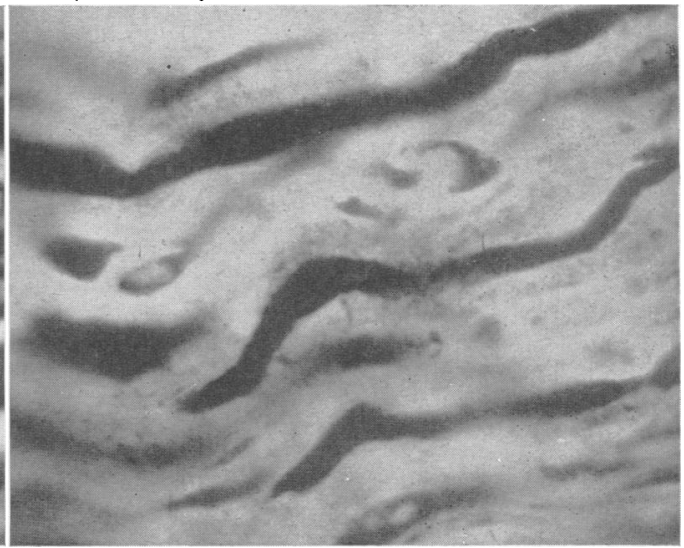

Fig. 12.-Axons of the peroneal nerve 27 hours after "degeneration" in vitro. Nerve excitable. (Gross Bielschowsky, $\times$ 750.) 


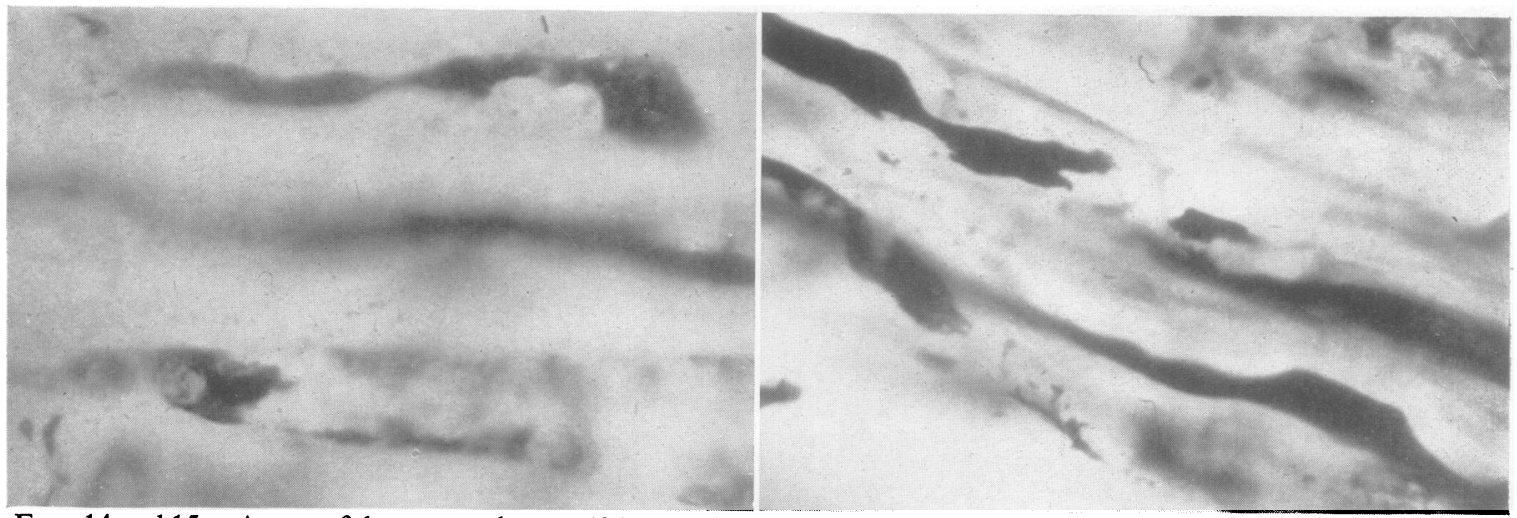

FIGS. 14 and 15.-Axons of the peroneal nerve 48 hours after " degeneration " in vitro. Nerve not excitable. Breakdown of the axons into irregular segments. (Gross Bielschowsky, $\times 750$.)

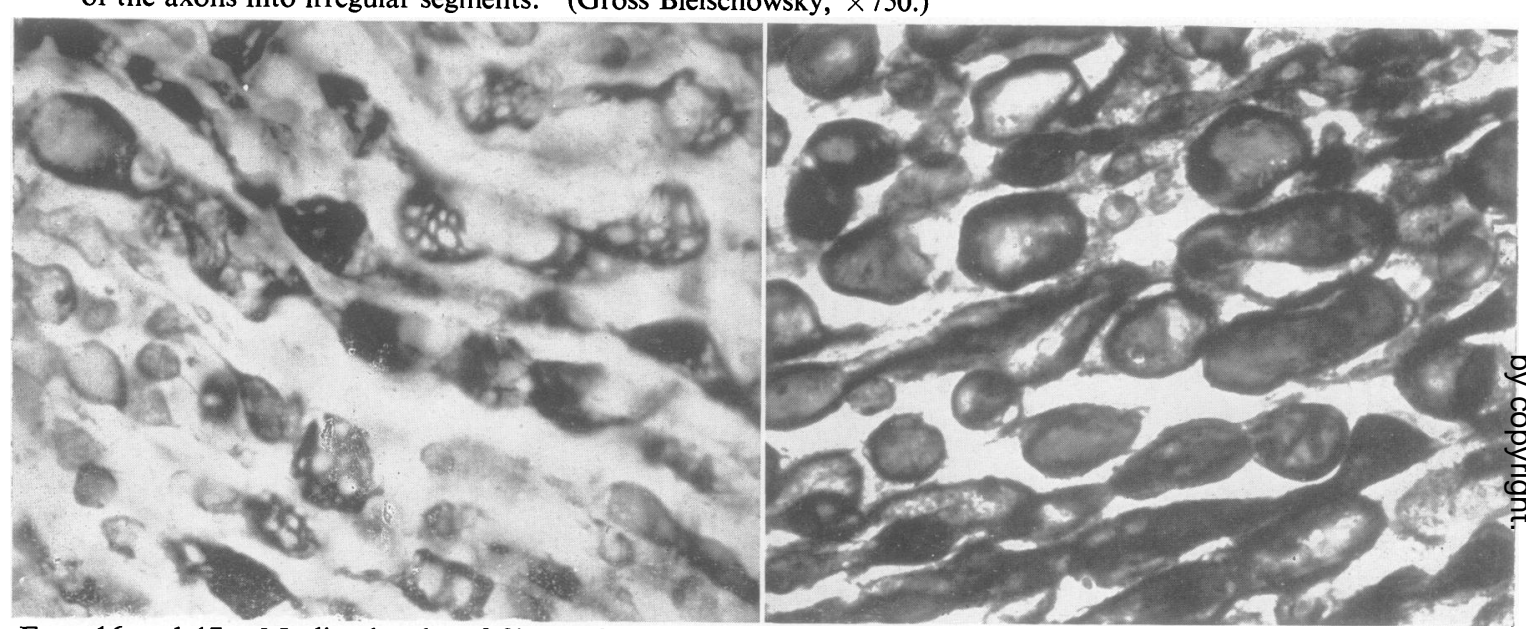

FIGs. 16 and 17.- Myelin sheaths of fibres of the peroneal nerve 48 hours after "degeneration" in vitro. They are breaking down into ovoids and globules, show a flaky appearance, and sometimes stain poorly. (Spielmeyer, $1 \times 380$.)

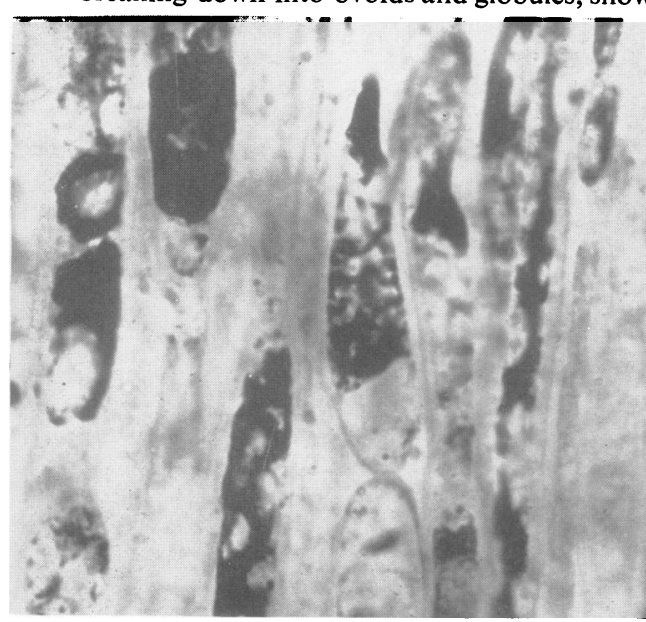

Fig. 18.-Axons in the peroneal nerve left to autolyse in the dead rabbit for five days at a temperature of $20^{\circ} \mathrm{C}$. The axons are breaking down into small segments and granules. (Gross Bielschowsky, $\times 750$.)

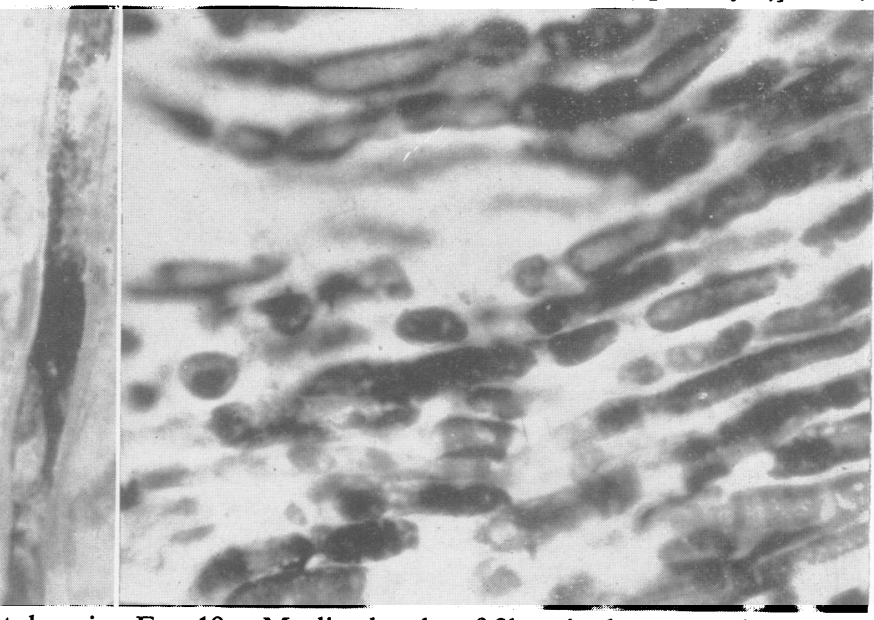

IG. 19.-Myelin sheaths of fibres in the peroneal nerve left to autolyse in the dead rabbit for five days at a temperature of $20^{\circ} \mathrm{C}$. They show bad staining and are breaking down into segments and ovoids. (Spielmeyer, $\times 380$.) 
The excitability of the nerves was then tested repeatedly at intervals of six hours. Failure of transmission of nerve impulses was recorded between 24 to 51 hours $(36 \pm 7)$. There is a considerable scatter of the data (much more than in degeneration in vivo), indicating interference by some uncontrolled factors. During degeneration the height of the action potential decreases progressively, but earlier than in nerve fibres degenerating in vivo. Conduction velocity decreases earlier and much more than in nerve fibres degenerating in vivo. This is shown in Fig. $X$ where conduction velocity is expressed as a percentage of that recorded immediately after excision of the nerve. A decrease of conduction velocity up to $42 \%$ was observed in one of the nerves tested. Fig. $Z$ shows the changes in one of the nerves tested. In this case the conduction velocity 26 hours after excision was $58 \%$ of that recorded at the beginning of the experiment. Two hours later the nerve was no longer excitable.

Similar experiments were performed with the sciatic nerve of rats. Failure of transmission of nerve impulses was observed 24 to 36 hours after excision of the nerves.

Histological Observations (Peroneal Nerve of Rabbits). Twelve hours after excision (action potential, +) the axons are at places swollen but their continuity is everywhere intact, while the myelin sheaths show no breakdown of continuity. Twenty-four hours after excision (action potential, + ) there is still continuity of axons, but they are considerably swollen with the formation of thin connecting bridges. The myelin sheaths have a flaky appearance, and in some of them there is already a definite breakdown of continuity. Twentyseven hours after excision (action potential, +) almost all of the axons are still in continuity (Fig. 12) but the myelin sheaths now show the formation of ovoid fragments which in some fibres are already very prominent (Fig. 13). Thirty-two hours after excision (action potential, +) examination shows a similar picture, but the formation of ovoids in the myelin sheaths is very marked. At this stage there is a considerable decrease of conduction velocity of the nerve fibres. Forty-eight hours after excision (action potential, -) all of the nerve fibres show loss of continuity. There is irregular breakdown of the axons, often with formation of fusiform swellings or small granules (Figs. 14 and 15). The myelin sheaths show no complete breakdown into globules and ovoids, but in many fibres the myelin shows a lack of staining reaction and has a flaky appearance (Figs. 16 and 17). Seventy-two hours after excision the axons and myelin sheaths show complete breakdown of continuity. The myelin in some fibres is broken down into small granules, the axons into clumps and granules. The morphological picture of degeneration in vitro is therefore similar to that observed in degeneration in vivo. Breakdown of the continuity of the axons can, similarly as in vivo, be correlated with failure in transmission of the nerve impulse. Before this occurs, however, there are definite changes in the myelin sheath. It appears that these rapidly progressing changes in the myelin sheaths are related to the considerable decrease of conduction velocity in nerve fibres degenerating in vitro. Such a decrease of conduction velocity is never observed in nerve fibres degenerating in vivo, which up to the time of failure of transmission of nerve impulses show few changes of their myelin sheaths. Otherwise, degeneration in vitro follows essentially the same morphological pattern as in vivo. There is breakdown of the axons and myelin sheaths with the typical formation of ovoids and globules. Failure of transmission of nerve impulses sets in earlier than in nerve fibres degenerating in vivo. It is however possible that better technical methods may slow down the rate of degeneration in vitro.

Degeneration of Nerve Fibres after Death.-After death the nerve fibres maintain excitability for a short time. At a temperature of $38^{\circ} \mathrm{C}$. failure of transmission of nerve impulses is recorded between two and a half and three and a half hours (four observations), and at a temperature of $20^{\circ} \mathrm{C}$. between three and four and a half hours after death (four observations).

At this interval the nerve fibres show no definite histological changes, and the loss of excitability is apparently due to anoxia. If the dead animals are kept at a temperature of $20^{\circ}$ the nerves still do not show marked changes 24 and 48 hours after death. There is still continuity of the axons and myelin sheaths, the axons are at places swollen and may show irregular or poor staining, while the myelin sheaths may show varicose swellings. Seventy-two hours after death most of the axons are still intact, but some show breakdown of continuity. The myelin sheaths show many varicose swellings and some ballooning ; in a very few fibres ovoid formation can be observed. Four days after death the axons show irregular outlines, and often poor staining, and some show breakdown of continuity into small segments or granules. The myelin sheaths show marked varicosities and ballooning, and also breaking down into short segments, ovoids, or granules. The myelin often stains pale blue and has a flaky appearance. A similar picture is seen five days after death. The axons are breaking down into small segments of irregular outlines or 
small granules (Fig. 18), the myelin sheaths show not only irregular staining and a flaky appearance, but also breakdown into ovoids and short segments (Fig. 19).

During post-mortem autolysis axons and myelin sheaths show therefore characteristic changes, which consist of a breakdown of continuity as occurs in vivo after nerve section. Bad staining and a flaky appearance of the myelin sheaths are absent during degeneration in vivo, but on the whole the breakdown of axons and myelin sheaths follows a similar pattern as in vivo.

\section{Discussion}

The physiological properties of nerves undergoing Wallerian degeneration have been studied repeatedly (Rosenblueth and Dempsey, 1939 ; Titeca, 1935 ; Parker, 1933 ; Erlanger and Schoepfle, 1946). Examination of these studies, however, reveals a number of inconsistencies. In the present study an attempt has been made to correlate morphological and physiological data and thus to determine the possible causes of the inconsistencies found in the reports of previous work. Titeca (1935) found during degeneration a striking exaggeration of fatigueability which appeared relatively early and developed in centrifugal direction. A centrifugal course of degeneration was also assumed by Rosenblueth and Dempsey (1939) and by Parker (1935). Erlanger and Schoepfle (1946), however, did not find a significant increase of fatigueability and did not confirm a centrifugal course of degeneration. They found failure of conduction at scattered loci increasing in frequency towards the periphery of the nerve. In our experiments we did not find any indication of a centrifugal course of degeneration. In vivo failure of conduction appeared to take place simultaneously in the proximal and distal half of the sectioned nerve trunk, and in vitro conduction failed at the ends of the nerve stump before the centre. This may be due to the protrusion and loss of material from the open ends of cut nerve fibres into the surrounding fluid. This apparently leads to an increase of interstitial fluid which is indicated by the softening and swelling of the ends of the nerve trunk. The increased interstitial fluid may act as a shunt for the recorded action potential and lead to lowering of its height at the ends of the nerve.

There are also some discrepancies when considering conduction velocity of degenerating nerve fibres. Erlanger and Schoepfle (1946) found in their preparations that conduction rates three days after nerve section were normal in the proximal parts but possibly declined slightly towards the periphery. This finding is, according to the authors, compatible with abrupt failure of conduction at random loci increasing in frequency towards the periphery. Rosenblueth and Dempsey (1939), however, found a small decline of conduction velocity during longer periods of degeneration, and they recorded an increase of conduction velocity towards the periphery. In our experiments we found a small but consistent decline of conduction velocity, the latter being the same in proximal and distal parts of the nerve. A progressive diminution of the spike potential magnitude, as measured from the area of the action potentials (Erlanger and Schoepfle, 1946) was found in all experiments (Rosenblueth and Dempsey, 1939; Erlanger and Schoepfle, 1946). We have made the same observation and our data correspond to those obtained by the other authors. Rosenblueth and Dempsey (1939) also found an increase of the spike potential magnitude as the stimulating cathode was moved peripherally and concluded that degeneration follows a centrifugal course. This was however not found by Titeca (1935) and Erlanger and Schoepfle (1946).

It is however difficult to interpret correctly data concerning the height or area of the action potential. The approximate relation between height of action potential and number of conducting nerve fibres is well known (Erlanger, Bishop, and Gasser, 1926), but this does not indicate an index for degenerating nerve fibres. We found continuity of all the axons intact at a time when the height of the action potential was markedly reduced. This may be due to the increase of water content in degenerating nerves (Mott and Halliburton, 1905), and consequently to the increased shunting of the action potentials recorded. Moreover, it is possible that the action potential of a single nerve fibre may decrease during degeneration. Such an assumption if verified would, of course, change the relation between the height of action potential and number of conducting nerve fibres during degeneration. Correlation of morphological and physiological data must therefore be undertaken carefully. The physiologically important observation is the time at which failure of transmission of nerve impulses can be recorded. This time was 71 to 78 hours after nerve section in our experiments on 1- to 2-year-old rabbits. Visible changes in the nerve fibres can however be observed before that time. Weddell and Glees (1941) found that changes become obvious within 12 hours after nerve section. It is clear that these changes, such as fusiform swelling or undulating outlines of the axons, may have some physiological significance but they do not abolish conduction of impulses. Failure of transmission of nerve impulses was observed once the 
majority of the nerve fibres showed breakdown of continuity of the axons. At this time no consistent changes in the myelin sheaths of nerve fibres degenerating in vivo could be observed. We have therefore used the loss of continuity of the axons as the index of degeneration in the morphological sense, and this index can be correlated with the physiological data. If, however, the term "signs of degeneration" is used in a vague sense only, misinterpretations when correlating morphological and physiological data are unavoidable. Erlanger and Schoepfle (1946) quote Weddell and Glees (1941) who find that all nerve fibres show signs of degeneration in some part of their course 48 hours after nerve section. On the basis of these morphological observations and of their findings that $25 \%$ of the fibres may still be responding almost, if not quite normally, 72 hours after nerve section, they conclude that conduction ceases only with disruption of the myelin sheath. Rosenblueth and Dempsey (1939) state that even when the structure of the nerve is grossly disrupted, its electrical excitability, spike potential, and conduction velocity may be practically normal. It is therefore necessary to define more exactly the term degeneration in the morphological sense. In our experiments we found that failure of transmission sets in when loss of continuity of the axons appears, even though no consistent changes are detectable in the myelin sheaths. This presumably explains the observation that irreversible loss of function appears quite suddenly (Rosenblueth and Dempsey, 1939 ; Erlanger and Schoepfle, 1946 ; Titeca, 1935).

However, it is clear that a completely reliable correlation of physiological and morphological data is possible only by a study of single fibres, and such a study should be possible. We have however been concerned mainly with the factors affecting the rate of degeneration. Such a study is complicated by the fact that not all fibres in a nerve trunk degenerate at the same rate. We found that sensory degenerate more quickly than motor nerve fibres and this agrees with the morphological findings of Moenkeberg and Bethe (1899) and those of Weddell and Glees (1941). Rosenblueth and Dempsey (1939) conclude on the basis of indirect evidence that the fastest fibres are the first to degenerate, but their conclusion is based on the assumption that degeneration follows a centrifugal course, and this has not been substantiated. In a few nerves they also found a normal $\mathrm{C}$ spike at a time when the $\mathrm{A}$ spike was considerably smaller than normal. Similarly, we found at a later stage of degeneration the $C$ spike to be larger than the A spike. It is possible that a number of unmedullated nerve fibres degenerate more slowly than the touch fibres (Weddell and
Glees, 1941). In any case, we did not observe failure in a motor nerve before that of a sensory nerve. We considered therefore (Gutmann and Holubár, 1949) that thickness of the myelin sheath may be the main factor in determining the rate of degeneration after nerve section, and that these findings may be useful when classifying nerve fibres according to their morphological and physiological characteristics. Some evidence for this assumption was afforded by experiments in which we "produced" thin nerve fibres, i.e., regenerating fibres and fibres in the central stump of a sectioned nerve trunk, and followed their rate of degeneration. Such nerve fibres degenerate more quickly, and it appears that during regeneration, i.e., with the increase of the diameter of the nerve fibres, the rate of degeneration decreases progressively. It will be necessary to study the time course of degeneration in other sets of nerve fibres, especially sympathetic nerve fibres, before general conclusions can be made.

A surprising observation is the finding that unconnected nerve fibres (in a twice-sectioned nerve trunk) do not lose excitability earlier than in a connected one, and that the action potential in such a nerve was always higher than in the connected (once-sectioned) nerve. We may therefore conclude that in some of the peroneal nerve fibres failure of transmission of nerve impulses sets in earlier in connected than in unconnected nerve fibres. That these fibres which lose excitability earlier are not necessarily motor fibres is suggested by the observation that conduction velocity which would be related to the quickest, i.e., motor nerve fibres, was always the same in both connected and unconnected nerve. We suggest that the proprioceptive nerve fibres especially are dropping out at a quicker rate in the connected nerve, and that this produces the smaller spike of the action potential of the connected nerve. It is probable that after section of a mixed nerve the proprioceptive nerve fibres are still being stimulated by the many changes of tension of the muscles which must still be operative. Such stimuli will of course not be active in fibres unconnected with their end organs, i.e., the muscle spindles. It has been shown that continuous stimulation of nerve fibres increases the rate of degeneration (Cook and Gerard, 1931), and it is therefore suggested that continuous stimulation of the proprioceptive nerve fibres results in quicker degeneration, and that this produces the smaller spike potential of a sectioned nerve connected with the end organs. It is also possible that the same factor, i.e., continuous stimulation of degenerating nerve fibres connected with their end organs, plays a part in the quicker rate of degeneration of skin sensory nerve fibres. 
It is therefore not surprising that the rate of degeneration is not significantly altered in a "free nerve graft". The method of recording failure of transmission of nerve impulses in these grafts enabled us to compare the rate of degeneration in auto-, homoeo-, and hetero-grafts. The time of failure of transmission of nerve impulses in homoeo-, and especially in hetero-grafts, could not be correlated with the morphological findings as was the case in autografts. In these grafts excitability ceased at a time when axons were still in continuity. Failure of transmission of nerve impulses was therefore due to other factors than the breakdown of the continuity of the axons. It is probable that the grafts act as antigens calling forward the formation of antibodies which destroy the graft. The quicker "degeneration" of homoeo- and heterografts seems to show the result of an immunobiological reaction as observed in homoeografts of skin which are destroyed by a reaction conforming in broad outline to that of active acquired immunity (Medawar, 1944 and 1945). However, the quick reaction without the usual latent period allows another explanation. Homoeo- and hetero-grafts produce a prompt and considerable cellular reaction and it is possible that this cellular reaction on the part of the host accomplishes the early destruction of the graft. Such an explanation would be in accordance with Loebs' theory (1930) of a local cellular mechanism. We cannot answer this question on physiological or histological grounds, but we may note that the " rate of degeneration" in homoeo- and hetero-grafts may give a quantitative test for immunobiological reactions, and may provide a means of testing the "denaturing" or "desensitizing" processes of the host.

In homoeografts " degeneration" (breakdown of axons and myelin sheaths) proceeds on the whole as in autografts. In heterografts many fibres persist, but breakdown of axons and myelin sheaths does occur though delayed and in an abnormal manner. This finding agrees with that of Macabruni (1911), but not with those of Merzbacher (1905) and Sanders and Young (1942) who did not find any signs of Wallerian degeneration in heterografts. In homoeoand much more pronouncedly in hetero-grafts, there is a quick pyknosis and often necrosis of the Schwann cells. The Schwann cells in heterografts do not undergo proliferation (Sanders and Young, 1942). The process in the Schwann cells apparently produces considerable connective tissue reaction and collagenization of the Schwann tubes, which will make later regeneration through this scarred tissue difficult or impossible.

The question whether Wallerian degeneration is a " vital" process and whether it takes place only in a medium of living tissue has been discussed repeatedly. Moenkeberg and Bethe (1899) concluded from their experiments that the process of Wallerian degeneration takes place only in nerves lying in a medium of living tissue, but Feiss and Cramer (1912) showed that nerves kept in Ringer solution showed "the classical signs of Wallerian degeneration" as seen in nerves of living animals. They did not, however, find a positive Marchi reaction, and there were no signs of nuclear activity. Clearly, the changes during degeneration in vitro can be compared with those in vivo only as long as the nerve can be kept alive. This will not be possible for 10 days, and a negative Marchi reaction cannot therefore serve as an argument for an assumption that degeneration in vitro differs from that occurring in vivo. In our experiments, failure of transmission of nerve impulses occurs not much earlier than in vivo. Morphologically the axons and myelin sheaths break up in the same manner. There is a quicker breakdown of the myelin sheaths taking place before breakdown of the axons and this process is apparently related to the considerable and quick decrease of conduction velocity. Changes in the myelin sheath apparently affect conduction velocity. The changes, in vivo, of the myelin sheaths which appear before failure of transmission of nerve impulses (breakdown of continuity of the axons) are relatively small, and correspondingly the decrease of conduction velocity is not marked. Morphologically, degeneration as indicated by break-up of the continuity of the axons and of the myelin sheaths, can be observed also during autolysis of nerves. Morphologically, degeneration, whether in vivo, in vitro, or under autolysis, follows a similar pattern. There is breakdown into globules, droplets, and ovoids. The nerve fibres degenerating in vitro, and especially under autolysis, show a flaky appearance, and later poor staining, but on the whole degeneration follows a similar morphological pattern.

Such observations lead to a discussion of the question, Why does a nerve fibre degenerate? Waller's (1852) original answer to this question implied that degeneration was due to the loss of impulses travelling along the nerve fibres. However, Cook and Gerard's (1931) and our findings indicate that stimulated nerve fibres degenerate at a quicker rate. Moreover, an unconnected, relatively inactive nerve fibre shows changes due to atrophy of inactivity, but will not undergo the rapid and apparently automatic breakdown of continuity of axons and myelin sheaths which is characteristic of degeneration following nerve section. The processes serving the quick transmission of nerve impulses cannot therefore be responsible for the maintenance of 
continuity of a nerve fibre. Muralt (1945) distinguishes between external and internal signals in nerve fibres, the latter serving the maintenance of reactivity (Betriebsbereitschaft und Betriebssicherheit). This term is a very vague one and unless explained more accurately seems to serve no more purpose than the equally vague term "trophic influence".

The factors responsible for the maintenance of the continuity of nerve fibres should also define the term "trophic influence". The "trophic influences" in nerve fibres may be somehow specific and different from those in other cells. It must be remembered that a nerve fibre may in fact be a very long ( 1 metre and more) cell process. The metabolism of nerve fibres is therefore the metabolism of long cells and may have some special characteristics. Gerard (1932) suggested that substances travelling from the cell nucleus into the fibre are involved in the problem of nerve degeneration. In this connexion it is interesting that the material injected below the perineurium moves distally in the endoneurial fluid in living and excised but not in dead nerve trunks (Weiss, Wang, Taylor, and Edds, 1945). It has also been observed that the material of axons is fluid and can flow freely from the ends of large axons of Cephalopods (Young, 1936) and that nucleoproteins pass continuously from the nucleus to the neurons (Hyden, 1943). It is therefore probable that during the whole life of the neuron substances are continuously passing along the nerve fibres. However, the passing down of substances will often only give an indication of certain, mainly enzymatic, processes going on in the nerve cell and the important task will be the definition of these processes which apparently maintain the continuity, in fact the socalled trophic conditions of the nerve fibres. A clear answer to this question is given by Young $(1944 ; 1945 ; 1945)$ who assumes that Wallerian degeneration in a severed nerve is produced by operation of forces of surface tension, and that the contents of the axons are normally under pressure from the nerve cell body. Nerve fibres and their sheaths are regarded as viscid liquid columns which become unstable once intra-axonal " growth pressures" are eliminated by section of a nerve trunk. Thus the pressure flow within the axon is thought to constitute the "trophic influence" which maintains the continuity of a nerve fibre. This theory is based especially on morphological observations on the pattern of the break-up of axons and myelin sheaths which appears to follow "with almost classical regularity the features of the separation of drops". The theory suggests that the myelin is a fluid acted on by surface tension and seems convincing in its simplicity. However, if a nerve fibre is maintained intact by pressure within its axon one would expect that the speed of breakdown would be the same under different conditions. Different factors, however (e.g. grafting), affect the rate of degeneration considerably. Moreover. it should be possible to prevent or delay degeneration by changing the osmotic pressure of the fluid surrounding the nerve. This however has so far not been demonstrated (Young, 1945).

It appears therefore that surface tension is not sufficient to explain completely the trophic influence which would maintain the integrity of a nerve fibre. The similarity with which degeneration proceeds in nerve fibres in vivo, in vitro, and under autolysis suggests the effect of a common process. We suggest that this common process is the autolysis occurring in the nerve fibre which finds its morphological expression in the breakdown of the axons and myelin sheaths. It is probable that forces of surface tension are operating during this breakdown of continuity, and affect the morphological pattern of degeneration, but the primary process leading to the changes following nerve section would be the "autolysis in vivo". The known enzyme complexes involved in autolysis are almost exclusively proteolytic ones and little seems to be known about the autolytic break-up of lipoids. The enzymatic processes proceeding during autolysis are essentially the same as those occurring in vivo. But in vivo all the enzymatic processes are continuously reversible and all the processes of breakdown are followed by processes of resynthesis. After nerve section certain substances will not pass to the peripheral stump and enzymatic processes will proceed unhampered without being followed by resynthesis and autolysis will result. During autolysis an increase of water content and liberation of phosphoric acid is observed (Bradley, 1938), and the same is the case during degeneration accompanying the breakdown of lipoids to fatty acids and phosphoric acid. This process also decreases the $p \mathrm{H}$ of the tissue, a factor which activates the hydrolysing proteolytic ferment kathepsin.

The assumption that degeneration is autolysis in vivo is so far only a working hypothesis, and further experiments will be necessary to substantiate it. Moreover, it must be noted that not all enzymatic processes in a sectioned nerve have the nature of an autolysis. During degeneration, for example, the formation of acethylcholine by cholineacetylase in the axon decreases, and 72 hours after nerve section loss of this enzyme activity amounts to $70 \%$ (Berman and Nachmansohn, 1946) and Wallerian degeneration is attended by a loss of $60 \%$ of true cholinesterase content (Sawyer, 
1946). However, it is possible that autolytic processes affect these enzymatic processes. For the main processes of breakdown of proteins and lipoids during degeneration irreversible enzymatic processes of autolysis seem to be responsible, and this " autolysis in vivo" follows the same morphological pattern as degeneration in vitro and that under autolytic conditions.

\section{Summary}

(1) Factors affecting the degeneration of peripheral nerve fibres after nerve section in rabbits were studied by recording the action potentials and correlating the results with the histological picture.

(2) In the peroneal nerve of adult rabbits transmission of nerve impulses ceases 71 to 78 hours after nerve section. Similar results were obtained in the sciatic nerves of rats and guinea-pigs. At this stage a breakdown in the continuity of almost all axons is observed.

(3) During degeneration the height of spike potentials decreases progressively. This decrease is considerable at a time when there is still continuity of all the axons.

(4) The decrease of the conduction velocity during degeneration is small, for even just before failure of transmission the decrease is only $13 \%$ of normal. At this time there are only very slight changes in the myelin sheaths.

(5) No differences in the rate of degeneration were found between the proximal and distal parts of the nerve stump. Degeneration appears to proceed simultaneously in the whole nerve stump.

(6) The rate of degeneration decreases with the age of animals.

(7) Failure of transmission sets in about 10 hours earlier in the sural than in the peroneal nerve. Thus the sensory nerve fibres degenerate more quickly than the motor ones, and this appears to be related to their thinner myelin sheaths.

(8) Regenerating nerve fibres and fibres in a central stump, 6 to 12 months after nerve section degenerate more quickly. These fibres have thin myelin sheaths. Thickness of the myelin sheaths is therefore probably an important factor affecting the rate of degeneration.

(9) Some unmyelinated fibres, however, appear to be more resistant to degeneration than the A fibres.

(10) In twice-sectioned nerves (i.e., nerves unconnected with their end organs) degeneration occurs at the same rate, the amplitude of the action potential being always higher than in connected ones (i.e., once-sectioned nerves). This is thought to be due to the effect of stimulation of proprioceptive nerve fibres in nerves connected with their end organs, on hastening degeneration.
(11) Completely isolated autotransplanted nerves show the same physiological and morphological characteristics as sectioned nerves left in situ.

(12) In homoeografts the failure of transmission of nerve impulses sets in after 44 to 48 hours, while in heterografts transmission fails about 15 hours after transplanting. The histological picture of homoeografts is similar to that of autografts. In heterografts some breakdown of axons and myelin sheaths does occur, but in most of the fibres no breakdown can be observed.

(13) Isolated nerves kept in vitro (Krebs solution with $\mathrm{O}_{2}+\mathrm{CO}_{2}$ supply at $38^{\circ}$ C.) " degenerate" (i.e., lose excitability) about 36 hours after excision. This failure of transmission of nerve impulses appears to be related to the breakdown of continuity of axons. Earlier and considerable changes in the myelin sheaths are apparently related to the quicker and greater decrease of conduction velocity (up to $40 \%$ ) in fibres degenerating in vitro.

(14) Nerves left in dead animals cease to conduct impulses in 2 to 4 hours, apparently because of anoxia, but histologically the axons and myelin sheaths show similar characteristic changes (breakdown) as in vivo.

(15) The question, Why does the nerve fibre degenerate? is discussed and it is suggested that the Wallerian degeneration is due to irreversible enzymatic processes, i.e., to autolysis in vivo.

\section{REFERENCES}

Berman, M., and Nachmansohn, D. (1946). Proc. Fed. Amer. Soc. exp. Biol., 5, 122.

Bethe, A. (1903). Allgemeine Anatomie und Physiologie des Nervensystems. Leipzig.

Bradley, H. C. (1938). Physiol. Rev., 18, 173.

Cajal, S. Ramón y. (1928). "Degeneration and Regeneration of the Nervous System." London.

Cook, D. D., and Gerard, R. W. (1931). Amer. J. Physiol., 97, 412.

Erlanger, J., Bishop, G. H., and Gasser, H. S. (1926). Ibid., 78, 537.

Erlanger, J., and Gasser, H. S. (1937). " Electrical Signs of Nervous Activity." Philadelphia.

Erlanger, J., and Schoepfle, G. M. (1946). Amer. J. Physiol., 147, 550.

Feiss, H. O., and Cramer, W. (1912). Proc. roy. Soc. B. 86, 119.

Gerard, R. W. (1932). Physiol. Rev., 12, 469.

Grundfest, H. (1940). Ann. Rev. Physiol., 2, 217.

Gutmann, E.: and Holubár, J. (1948). Cas. Lék. čes., 87,648 .

-—, - - (1949). Nature, 163, 328.

__, and Sanders, F. K. (1943). J. Physiol., 101, 489.

Holubár, J. (1949). Biol. Listy, 30, 26.

Hydén, H. (1943). Acta. physiol. scand., 6, suppl. 17, 1.

Johnson, A. C., McNabb, A. R., and Rossiter, R. J. (1949). Nature, 164, 108.

Loeb, L. (1930). Physiol. Rev., 10, 547.

Lundberg, A. (1948). Acta. physiol. scand., 15, suppl. 50.

Maccabruni, F. (1911). Folia Neuro-Biologica, 5, 598.

Medawar, P. B. (1944). J. Anat., Lond., 78, 176. 
Medawar, P. B. (1945). J. Anat., Lond., 79, 157.

Merzbacher, L. (1905). Z Zbl. Neurol., 24, 150.

Moenckeberg, G., and Bethe, A. (1899). Arch. mikr. Anat., 54, 135.

Mott, F. W., and Halliburton, W. D. (1901). Philos. Trans. B., 194, 437.

Muralt, A. v. (1945). "Die Signaluebermittlung im Nerven." Basel.

- and Schulthess, G. v. (1944). Helv. physiol. pharmacol. Acta., $2,435$.

Nageotte, J. (1922). "L'organisation de la Metière dans ses Rapports avec la Vie." Paris.

Parker, G. H. (1933). Amer. J. Physiol., 106, 398.

Prickett, C. O., and Stevens, C. (1939). Amer. J. Pathol., 15, 241.

Rosenblueth, A., and Dempsey, E. W. (1939). Amer. J. Physiol., 128, 19.
Sanders, F. K., and Young, J. Z. (1942). J. Anat., Lond., 76, 143.

Sawyer, C. H. (1946). Amer. J. Physiol., 146, 246.

Scharrer, E. and B. (1945). Physiol. Rev., 25, 171.

Titeca, J. (1935). Arch. int. Physiol., 41, 1.

Waller, A. (1852). C. R. Soc. Biol., Paris, 34, 582.

Weddell, G., and Glees, P. (1941). J. Anat., Lond., 76, 65 .

Weiss, P., Wang, H., Taylor, A. C., and Edds, M. V. (1945). Amer. J. Physiol., 143, 521.

Young, J. Z. (1936). Proc. roy. Soc. B., 121, 319. (1944). Nature, 154, 521. (1945). Ibid., 156, 132.

(1945). "The History of the Shape of a Nerve Fibre in Essays on Growth and Form presented to D'Arcy W. Thompson." Oxford Univ. Press, London. P. 41. 